\title{
Actualización: ¿Cuán apropiadamente estamos rastreando osteoporosis antes de los 65 años?
}

How appropiatelly are we screening osteoporosis before 65?

María de las Nieves Ganiele, Georgina Bonavita , Sergio Terrasa y Karin Kopitowski

\begin{abstract}
Resumen
Más allá de las fracturas causadas por un trauma mínimo y que definen en forma contundente la presencia de osteoporosis, en 1994 la Organización Mundial de la Salud (OMS) propuso criterios diagnósticos de osteoporosis (OP) basados en la determinación de la densidad mineral ósea (DMO), lo que permite pensar a la osteoporosis como uno de los factores de riesgo de fracturas. Para las mujeres adultas, el punto de corte es de 2,5 desviaciones estándar (DS) por debajo del promedio observado a la edad en la que se alcanza la masa ósea máxima.

Las principales guías de práctica clínica acuerdan en iniciar el rastreo de OP a los 65 años en mujeres sin factores de riesgo de padecerla, recomendando comenzar antes sólo cuando pertenecen a una subpoblación de riesgo aumentado por la presencia de al menos un factor de riesgo de OP (antecedente de fractura de cadera en un familiar de primer grado, fractura osteoporótica previa, uso de corticoides sistémicos por al menos tres meses, abuso de alcohol, tabaquismo activo, bajo índice de masa corporal, artritis reumatoidea o causas de osteoporosis secundaria); o por la presencia de un riesgo de fractura a diez años superior a 9,3\% calculado con la regla de predicción clínica desarrollada por la OMS denominada FRAX ${ }^{\mathrm{TM}}$ y que requiere información sobre la mayoría de los predictores previamente mencionados.
\end{abstract}

\begin{abstract}
Beyond fractures caused by minimum trauma and that strongly define the presence of osteoporosis, in 1994 World Health Organization (WHO) proposed diagnostic criteria of osteoporosis (OP) based on the determination of bone mineral density (BMD), which allows to think about osteoporosis as one of the risk factors for fractures. For adult women, a cut-off point is 2.5 standard deviations (DS) below the average observed at the age in which the maximum bone mass is reached.

Main clinical practice guides agree to initiate screeening of OP at the age of 65 in women without OP risk factors. They advice to begin before that age only when the woman is included in a subpopulation of increased risk of OP defined by the presence of at least one risk factor for OP (history of hip fracture in a first-degree relative, previus osteoporotic fracture, use of systemic steroids for at least three months, alcohol abuse, active smoking, low body mass index, rheumatoid arthritis or causes of secondary osteoporosis); or defined by the presence of a risk of fracture score over $9.3 \%$ at ten years, calculated with the clinical prediction rule for osteoporosis called FRAX TM with requires information of most of the previously mentioned predictors.
\end{abstract}

Palabras clave: fractura osteoporótica, osteoporosis, rastreo, estratificación del riesgo. Key words: osteoporotic fracture, osteoporosis, screening, risk stratification.

Ganielle M, Bonavita G, Terrasa S, Kopitowski K. ¿Cuán apropiadamente estamos rastreando osteoporosis antes de los 65 años?. Evid. Act. Pract. Ambul. Abr-Jun 2012;15(2): 71-75.

\section{Algunas definiciones y descripción del problema}

La osteoporosis (OP) es una condición clínica caracterizada por una baja masa ósea y por un deterioro de la microarquitectura del tejido óseo, con un consiguiente aumento de la fragilidad ósea y la susceptibilidad a las fracturas'. Puede afectar a ambos sexos, pero las mujeres están más predispuestas luego de la menopausia debido a que la disminución en la producción de estrógenos acelera la pérdida ósea.

\section{Fracturas patológicas por osteoporosis}

Una fractura osteoporótica por fragilidad es definida por la Organización Mundial de la Salud (OMS) como aquella causada por un trauma leve (fuerza equivalente a una caída de propia altura o menos) y que resulta de una fuerza o torsión que se ejerce sobre el hueso, que sería insuficiente para fracturar hueso normal.

Las fracturas osteoporóticas ocurren principalmente en la columna vertebral, cadera (fémur proximal) y muñeca (radio distal). Pueden causar dolor crónico, síntomas psicológicos como depresión o menor autoestima, discapacidad severa, pérdida de la independencia y se asocian a disminución de la calidad y la expectativa de vida. ${ }^{2,3 \neq}$

\section{Osteoporosis como factor de riesgo}

Por otro lado y más allá de las fracturas causadas por osteoporosis, en 1994 un panel de expertos de la Organización Mundial de la Salud (OMS) propuso criterios diagnósticos de OP para el uso clínico basados en la determinación de la densidad mineral ósea (DMO), lo que permite pensar a la osteoporosis como un factor de riesgo. Estos criterios se fundamentan en múltiples estudios prospectivos que demuestran que el riesgo de fracturas de tipo osteoporótico aumenta progresiva y continuamente en la medida que la DMO disminuye (correlación negativa).

Para las mujeres adultas, el punto de corte es de 2,5 desviaciones estándar (DS) por debajo del promedio observado en la población sana de 30 años (edad en la que se alcanza la masa ósea máxima) y se reporta como un puntaje $\mathrm{T}$ (en inglés $\mathrm{T}$ score) menor o igual a $-2,5^{\$ \S}$. \footnotetext{
\# En particular la fractura de cadera genera un exceso de mortalidad del 10 al $20 \%$ al año, aproximadamente $20 \%$ de los pacientes requieren atención domiciliaria de enfer-
mería a largo plazo y solo $40 \%$ recupera totalmente el nivel de independencia previo. La mortalidad también aumenta con las fracturas vertebrales, otras de sus complicaciones incluyen dolor lumbar, pérdida de altura y cifosis con alteraciones posturales. La fractura de muñeca es menos incapacitante pero puede interferir en actividades de la vida diaria.

${ }^{\$}$ Con objeto de establecer un umbral para medidas preventivas para atenuar las consecuencias de este factor de riesgo descripto, muchos expertos coinciden que el punto de corte sea una desviación estándar (DS) debajo del promedio de la población sana de 30 años, vale decir un T-score menor a -1, que se define como osteopenia.
}

${ }^{\ddagger}$ Servicio de Medicina Familiar y Comunitaria de Hospital Italiano de Buenos Aires (HIBA) maria.ganiele@ @ospitalitaliano.org.ar

${ }^{\S}$ Alumna de la Carrera de Medicina del Instituto Universitario Hospital Italiano de Buenos Aires.

** Servicio de Medicina Familiar y Comunitaria del HIBA y Departamento de Salud Pública del Instituto Universitario HIBA.

+ Servicio de Medicina Familiar y Comunitaria del HIBA y Departamento de Medicina del Instituto Universitario HIBA. 


\section{Repercusión epidemiológica de la osteoporosis}

Se estima que la OP afecta a 200 millones de personas en todo el mundo***4+t+5,6,7

En un estudio realizado en cinco países de Latinoamérica ${ }^{\ddagger \neq}$, la prevalencia de fracturas vertebrales en mujeres mayores de 50 años de edad fue de alrededor del $15 \%$; mientras que en Argentina la prevalencia de osteopenia en mujeres mayores de 50 años es del $50 \%$ y la de OP, del $25 \%{ }^{9,10 s \$ s}$.

La repercusión pública de la carga de enfermedad asociada a esta condición clínica es motivo de controversia, existiendo múltiples evaluaciones económicas de su impacto****11tttt y de las intervenciones para prevenirla o tratarla. ${ }^{12}$ Por ejemplo, Johnell y col. estimaron que las fracturas osteoporóticas representan el $0,83 \%$ de la carga mundial de enfermedades no transmisibles ${ }^{13 \neq+\neq 14 ;}$; mientras que Ström y col. calcularon que la carga económica de las fracturas osteoporóticas excede a la generada por la del accidente cerebrovascular, la esclerosis múltiple, la enfermedad de Parkinson y la migraña, y que sería similar a la de la artritis reumatoidea ${ }^{15}$.

\section{Herramientas disponibles para su rastreo}

La DMO se utiliza como una valoración de la resistencia ósea, siendo el método más utilizado para su medición la absorciometría dual de rayos $\mathrm{X}$ (en inglés: dual energy $\mathrm{X}$-ray absorptiometry o "DXA"). El equipo que utiliza la técnica densitométrica DXA, comúnmente llamado densitometría ósea (DO), de la columna lumbar anteroposterior y fémur, es el más ampliamente usado en el mundo por sus favorables características operativas, su reproducibilidad, y la muy baja dosis de radiación que implica ${ }^{16}$. Sin embargo no es totalmente preciso ya que puede subestimar o sobreestimar la DMO en un 20 a $50 \%{ }^{17}$.

\section{Recomendaciones de rastreo}

Respecto de la edad de comienzo del rastreo de la osteoporosis, las principales guías de práctica clínica acuerdan que sea a los 65 años en mujeres sin factores de riesgo de padecerla ${ }^{\$ \$ 18,19,20}$. En la tabla 1 se resumen los principales puntos de las guías de Estados Unidos ${ }^{\star * *}$, Inglaterra, Canadá y Argentina.

Tabla 1. Principales guías de práctica clínica que se han expedido sobre pautas de rastreo de la osteoporosis como factor de riesgo.

\begin{tabular}{|c|c|c|c|}
\hline \multirow[b]{2}{*}{ Guía } & \multicolumn{2}{|c|}{$\begin{array}{l}\text { Edad a la que recomiendan solicitar el } \\
\text { rastreo con densitometria ósea }\end{array}$} & \multirow[b]{2}{*}{ Factor de riesgo considerados por la cada guía de práctica clínica } \\
\hline & $\begin{array}{c}\text { En ausencia de } \\
\text { factores de riesgo }\end{array}$ & $\begin{array}{c}\text { En presencia de al menos un } \\
\text { factor de riesgo }\end{array}$ & \\
\hline $\begin{array}{l}\text { U.S. Preventive Services } \\
\text { Task Force: } \\
\text { Screening for } \\
\text { osteoporosis } \\
\text { recommendation } \\
\text { statement } 2011 \text { (EE.UU) } \\
\end{array}$ & $\begin{array}{l}\text { Mujeres luego de } \\
\text { los } 65 \text { años }\end{array}$ & $\begin{array}{l}\text { Mujeres entre los } 50 \text { y los } 64 \\
\text { años }\end{array}$ & $\begin{array}{l}\text { Riesgo de fractura a diez años sea mayor o igual a } 9,3 \% \text { de acuerdo a los resultados de la } \\
\text { calculadora de riesgo FRAXTM }\end{array}$ \\
\hline $\begin{array}{l}\text { American Association of } \\
\text { Clinical Endocrinologists } \\
\text { Medical: Guidelines for } \\
\text { the prevention and } \\
\text { treatment of } \\
\text { osteoporosis } 2010 \\
\text { (EE.UU) }\end{array}$ & $\begin{array}{c}\text { Mujeres luego de } \\
\text { los } 65 \text { años }\end{array}$ & Mujeres post-menopáusicas & $\begin{array}{l}\text { - Antecedente de fracturas ante mínimos traumatismos luego de los } 40 \text { ó } 45 \text { años de edad } \\
\text { - Presencia de osteopenia identificada radiográficamente } \\
\text { - Haber realizado un tratamiento prolongado (más de tres meses) con glucocorticoides } \\
\text { sistémicos } \\
\text { - Bajo peso (IMC menor de } 20 \mathrm{~kg} / \mathrm{m} 2 \text { ) } \\
\text { - Antecedente de familiar con fractura osteoporótica } \\
\text { - Menopausia temprana } \\
\text { - Tabaquismo activo } \\
\text { - Consumo excesivo de alcohol } \\
\text { - Osteoporosis secundaria (por ej. artritis reumatoidea) } \\
\text { - Factores de riesgo para caídas (problemas neurológicos como enfermedad de Parkinson, } \\
\text { demencia, accidente cerebrovascular, problemas de la visión, uso de medicación sedativa o } \\
\text { hipnótica, factores del ambiente, antecedente de caída previa, etc.) }\end{array}$ \\
\hline
\end{tabular}

\footnotetext{
*** Afecta aproximadamente a una décima parte de las mujeres mayores de 60 años, a una quinta parte de las mujeres mayores de 70 años, a dos quintas partes de las mujeres mayores de 80 años y a dos tercios de las de 90 años.

"It En Canadá la OP afecta a 1.4 millones de habitantes, principalmente mujeres postmenopáusicas. En Estado Unidos en el año 2002 , basados en datos de la Encuesta Nacional de salud y nutrición III, se estimó que 10 millones de norteamericanos ya tenían OP ( $80 \%$ de ellos eran mujeres) y se cree que esta cifra se elevará a 14 millones para 2020. Por otro lado, en España aproximadamente 2 millones de mujeres tienen OP y su prevalencia es de 26,1\% en mayores de 50 años.

\#\#rasil, Argentina, Colombia, Méjico y Puerto Rico.

${ }^{\$ \$}{ }_{5}$ Se proyecta en Argentina que para 2050, 5,24 millones mujeres tendrán osteopenia y 2,62 millones, osteoporosis.

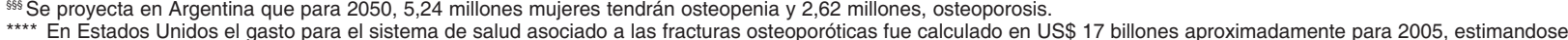
que por el creciente envejecimiento de la población el número de fracturas de cadera y su costo asociado podría duplicarse o incluso triplicarse para el año 2040.

$\mathrm{t+t}$ Un reporte realizado en el año 2011 en Europa en asociación con la International Osteoporosis Fundation y la European Federation of Pharmaceutical Industry Associations acerca de la carga de esta enfermedad revela que el costo total para los países más importantes del continente (Alemania, Francia, Inglaterra, España, Italia y Suecia), incluyendo el tratamiento farmacológico preventivo, es de $€ 30.7$ billones (3,5\% del gasto total en salud), siendo la fractura de cadera responsable del $54 \%$ del mismo.

$\ddagger \ddagger \ddagger$ Vale destacar que para algunos expertos esta condición clínica genera un importante impacto para la salud pública por su elevada prevalencia, la morbi-mortalidad asociada a sus complicaciones (fracturas por fragilidad) y los gastos directos e indirectos de su atención.

s\$ş Järvinem T. sostiene que idealmente y en condiciones de una adherencia poco realista del $70 \%$, sería necesario rastrear con DO a 731 mujeres de 65 a 69 años, e idealmente, tratar a 88 de ellas con bifosfonatos orales (esto sería, aquellas con OP) durante cinco años para prevenir una fractura de cadera (entre los 70 y los 74 años estos números son 254 y 51 , respectivamente).

números son 254 y 51, respectivamente).
} 


\begin{tabular}{|c|c|c|c|}
\hline $\begin{array}{l}\text { National Osteoporosis } \\
\text { Foundation: Clinician's } \\
\text { guide to prevention and } \\
\text { treatment of } \\
\text { osteoporosis } 2010 \\
\text { (EE.UU) }\end{array}$ & $\begin{array}{l}\text { Mujeres luego de } \\
\text { los } 65 \text { años } \\
\text { Hombres luego de } \\
\text { los } 70 \text { años }\end{array}$ & $\begin{array}{l}\text { Mujeres postmenopáusicas y } \\
\text { hombres de } 50 \text { a } 69 \text { años. }\end{array}$ & $\begin{array}{l}\text { - Antecedente de fractura de cadera en familiar de primer grado } \\
\text { - Tabaquismo activo } \\
\text { - Consumo excesivo de alcohol ( } \geq 3 \text { “tragos"/día) } \\
\text { - Bajo peso ( } \mathrm{IMC}<20 \mathrm{~kg} / \mathrm{m}^{2} \text { ) } \\
\text { - Tratamiento oral con glucocorticoides (más de } 5 \mathrm{mg} / \text { día de prednisona durante más de tres } \\
\text { meses } \\
\text { - Antecedente propio de fractura osteoporótica } \\
\text { - Artritis Reumatoide } \\
\text { - Osteoporosis secundaria }\end{array}$ \\
\hline $\begin{array}{l}\text { North American } \\
\text { Menopause Society: } \\
\text { Guidelines for screening } \\
\text { and treatment of } \\
\text { osteoporosis } 2010 \\
\text { (EE.UU) }\end{array}$ & $\begin{array}{l}\text { Mujeres luego de } \\
\text { los } 65 \text { años }\end{array}$ & $\begin{array}{l}\text { Mujeres postmenopáusicas } 0 \\
\text { mayores de } 50 \text { años }\end{array}$ & $\begin{array}{l}\text { - Fracturas por fragilidad luego de la menopausia } \\
\text { - Bajo peso (IMC menor a } 21 \mathrm{~kg} / \mathrm{m}^{2} \text { ) } \\
\text { - Antecedente de fractura de cadera en familiar de primer grado } \\
\text { - Tabaquismo activo } \\
\text { - Artritis reumatoidea } \\
\text { - Consumo diario de alcohol mayor a dos "tragos" /diarios } \\
\text { - Causas secundarias de pérdida ósea (hiperparatiroidismo, hipertiroidismo y anorexia nerviosa) } \\
\text { o consecutivas a intervenciones médicas: uso diario presente o pasado de esteroides durante } \\
\text { más de seis meses, uso de anticoagulantes (warfarina o heparina), o de anticonvulsivantes }\end{array}$ \\
\hline $\begin{array}{l}\text { Clinical practice } \\
\text { guidelines for the } \\
\text { diagnosis } \\
\text { and management of } \\
\text { osteoporosis in Canada } \\
2010\end{array}$ & $\begin{array}{l}\text { Ambos sexos } \\
\text { luego de los } 65 \\
\text { años }\end{array}$ & $\begin{array}{l}\text { Mujeres menopáusicas y } \\
\text { hombres de } 50 \text { a } 64 \text { años } \\
\text { Adultos menores de } 50 \text { años }\end{array}$ & $\begin{array}{l}\text { - Fracturas por fragilidad después de los } 40 \text { años } \\
\text { - Tratamiento prolongado con glucocorticoides } \\
\text { - Uso de otras medicaciones asociadas a mayor riesgo de disminución de la masa ósea } \\
\text { (inhibidores de la aromatasa, terapia de deprivación de andrógenos) } \\
\text { - Antecedente de fractura de cadera en familiar de primer grado } \\
\text { - Fractura vertebral u osteopenia identificada por radiografía } \\
\text { - Consumo excesivo de alcohol } \\
\text { - Tabaquismo activo } \\
\text { - Peso menor a } 60 \mathrm{~kg} \text { o pérdidas de peso que resulten en tener actualmente un peso de menor al } \\
\text { 90\% del que se tenía a los } 25 \text { años de edad (pérdida que supera el 10\%) } \\
\text { - Artritis reumatoidea } \\
\text { - Osteoporosis secundaria } \\
\text { - Hipogonadismo o menopausia precoz (antes de los } 45 \text { años) }\end{array}$ \\
\hline $\begin{array}{l}\text { National Institute for } \\
\text { Health and Clinical } \\
\text { Excellence: Osteoporosis } \\
\text { fragility fracture risk } \\
\text { clinical guideline } 2012 \\
\text { (Inglaterra) }\end{array}$ & $\begin{array}{l}\text { Mujeres luego de } \\
\text { los } 65 \text { años y } \\
\text { hombres luego de } \\
\text { los } 75\end{array}$ & $\begin{array}{l}\text { Mujeres de } 51 \text { a } 64 \text { años y } \\
\text { hombres de } 51 \text { a } 75\end{array}$ & $\begin{array}{l}\text { - Fractura por fragilidad previa } \\
\text { - Estar usando o haber usado glucocorticoides orales } \\
\text { - Historia de caídas } \\
\text { - Antecedente de fractura de cadera en un familiar de primer grado } \\
\text { - Osteoporosis secundaria } \\
\text { - Bajo peso (IMC menor a } 18,5 \mathrm{~kg} / \mathrm{m}^{2} \text { ) } \\
\text { - Tabaquismo activo (más de } 10 \mathrm{cigarrillos} / \text { día) } \\
\text { - Consumo excesivo de alcohol (más de cuatro unidades/día) }\end{array}$ \\
\hline $\begin{array}{l}\text { Consenso de la Sociedad } \\
\text { Argentina de } \\
\text { Osteoporosis y la } \\
\text { Asociación Argentina de } \\
\text { Osteología y } \\
\text { Metabolismo Mineral: } \\
\text { Guías para Diagnóstico, } \\
\text { Prevención y Tratamiento } \\
\text { de la Osteoporosis } 2007 \\
\text { (Argentina) }\end{array}$ & $\begin{array}{c}\text { Mujeres luego de } \\
\text { los } 65 \text { años y } \\
\text { hombres luego de } \\
\text { los } 70\end{array}$ & Mujeres menores de 65 años & $\begin{array}{l}\text { - Historia personal de fracturas } \\
\text { - Antecedente de fractura en familiar de primer grado } \\
\text { - Osteoporosis secundaria } \\
\text { - Menopausia precoz (antes de los } 40 \text { años) o quirúrgica (antes de los } 45 \text { ) } \\
\text { - Carencia de estrógenos en la premenopausia } \\
\text { - Delgadez (IMC menor a } 20 \mathrm{~kg} / \mathrm{m}^{2} \text { ) } 0 \text { trastornos de la conducta alimentaria } \\
\text { - Ingesta de corticoides u otras drogas (hormona tiroidea a dosis supresivas de la tirotrofina, } \\
\text { análogos de la hormona liberadora de gonadotrofinas, antiandrógenos, inhibidores de la } \\
\text { aromatasa, anticonvulsivantes, anticoagulantes, furosemida, tiazolidinedionas, inhibidores de la } \\
\text { bomba de protones, litio) } \\
\text { - Trasplante de órganos } \\
\text { - Tabaquismo (más de diez } 10 \text { cigarrillos/día) } \\
\text { - Amenorrea primaria o secundaria } \\
\text { - Inmovilización prolongada (mayores a tres meses) } \\
\text { - Bajo consumo de calcio }\end{array}$ \\
\hline
\end{tabular}

- Antecedente de fractura de cadera en familiar de primer grado

- Bajo peso $\left(\mathrm{IMC}<20 \mathrm{~kg} / \mathrm{m}^{2}\right)$

- Tratamiento oral con glucocorticoides (más de $5 \mathrm{mg} /$ día de prednisona durante más de tres

- Artritis Reumatoide

- Osteoporosis secundaria

- Bajo peso (IMC menor a $21 \mathrm{~kg} / \mathrm{m}^{2}$ )

- Antecedente de fractura de cadera en familiar de primer grado

Tabaquismo activo

- Causas secundarias de pérdida ósea (hiperparatiroidismo, hipertiroidismo y anorexia nerviosa) - Constivas a intervenciones médicas: uso diario presente o pasado de esteroides durante

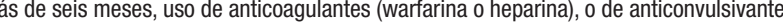

(inhibidores de la aromatasa, terapia de deprivación de andrógenos)

$90 \%$ del que se tenía a los 25 años de edad (pérdida que supera el $10 \%$ )

- Artritis reumatoidea

opausia precoz (antes de los 45 años)

- Estar usando o haber usado glucocorticoides orales

- Historia de caídas

- Osteoporosis secundaria

- Bajo peso (IMC menor a $18,5 \mathrm{~kg} / \mathrm{m}^{2}$ )

- Tabaquismo activo (más de 10 cigarrillos/día)

- Historia personal de fracturas

- Osteoporosis secundaria

- Menopausia precoz (antes de los 40 años) o quirúrgica (antes de los 45

encia de estrógenos en la premenopausia

- Ingesta de corticoides u otras drogas (hormona tiroidea a dosis supresivas de la tirotrofina, análogos de la hormona liberadora de gonadotrofinas, antiandrógenos, inhibidores de la

bomba de protones, litio)

- Trasplante de órganos

- Tabaquismo (más de diez 10 cigarrillos/día)

- Inmovilización prolongada (mayores a tres meses)

- Bajo consumo de calcio

FR: factores de riesgo; DO: densitometría ósea, IMC: índice de masa corporal.

Respecto de las mujeres postmenopáusicas menores de 65 años, estas mismas guías solo recomiendan que sean sometidas a DO de rastreo cuando pertenecen a una subpoblación de riesgo aumentado. Hay diferentes formas de definir a esta subpoblación:

a) Por la presencia de al menos un factor de riesgo de los siguientes: antecedente de fractura de cadera en un familiar de primer grado, fractura osteoporótica previa, uso de corticoides sistémicos por al menos tres meses, abuso de alco- hol, tabaquismo activo, bajo índice de masa corporal, artritis reumatoidea $u$ osteoporosis secundaria..$^{t+t t+}$ y b) Por la presencia de un riesgo de fractura a diez años superior a 9,3\%: esta población se define de acuerdo a los resultados de una regla de predicción clínica desarrollada por la OMS y denominada FRAX ${ }^{\mathrm{TM} 21,22,23}$ (recomendación tipo B) ${ }^{24}$ y que como insumo incluye información sobre la mayoría de los predictores previamente mencionados ${ }^{\sharp \neq \neq \neq \neq \ddagger}$. Ver figura 1.

ttrtt North American Menopause Society 2010 Guidelines for screening and treatment of OP, 2010 clinical practice guidelines for the diagnosis and management of OP in Canada, 2007 Guía para diagnóstico, prevención y tratamiento de la OP Soc. Argentina de OP y Asociación Argentina de Osteología y Metabolismo Mineral.

\#\#\#¥ La calculadora FRAXTM considera las características epidemiológicas locales de diferentes países (incluyendo Argentina, aunque no se hayan obtenido de un relevamiento de nivel nacional), le otorga a cada factor un coeficiente independiente que refleja su propio peso y que funciona con o sin datos sobre la DMO en el cuello femoral. Se utiliza para determinar qué individuos exceden el umbral de riesgo, sirve para hombres y mujeres de 40 a 90 años. Se considera a una mujer de raza blanca de 65 años sin otros factores de riesgo como el caso de base, obteniendo un riesgo a 10 años para cualquier fractura osteoporótica de $9,3 \%$. 
Figura 1. Regla de predicción clínica de uso libre propuesta por la Organización Mundial de la Salud que permite estimar el riesgo de fractura osteoporótica a diez años sobre la base de variables clínicas de fácil obtención en el consultorio.

Por favor responda las preguntas siguientes para calcular la probabilidad de fracturas a diez años sin DMO o con DMO

\begin{tabular}{|c|c|c|c|c|}
\hline \multicolumn{3}{|c|}{ Pais: Reino Unido Nombre/lD: } & \multicolumn{2}{|c|}{ Sobre los Factures de riesgo (i) } \\
\hline \multicolumn{3}{|c|}{ Cuestionario: } & 10. Ostcoporosis Secundaria & - No $\cup \mathrm{si}$ \\
\hline \multicolumn{3}{|c|}{1 Edad (antre $40-90$ arios) o techa ca nacimiento } & 11. Alcohol, 3 o mas dosis por dla & - Na U Si \\
\hline \multirow[t]{2}{*}{ Edad } & \multicolumn{2}{|c|}{ Facha de Nacimento: } & 12 cNo de Cuelo Femoral & \\
\hline & $m \square$ & 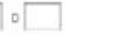 & Seleccione DXA * & \\
\hline $2 \operatorname{Sen} 0$ & Hambre & Mujer & Borrat & Calcular \\
\hline \multicolumn{3}{|c|}{3 Peso (ha) } & & \\
\hline \multicolumn{2}{|c|}{ 4. Estatura (cm) } & & & \\
\hline \multicolumn{2}{|c|}{5 Fractura Previa } & - Na Usi & & \\
\hline \multicolumn{2}{|c|}{ 6. Padres con tracthar de cadera } & - No $\circlearrowleft 1$ & & \\
\hline \multicolumn{2}{|c|}{ 7. Fumador Actwo } & - No Usi & & \\
\hline \multicolumn{2}{|c|}{ B Glucoconticoides } & - No Usi & & \\
\hline \multicolumn{2}{|c|}{ 9. Artritis Reumatoide } & - No Usi & & \\
\hline
\end{tabular}

Fuente: FRAX. Herramienta de Evaluación de Riesgo de Fractura desarrollada por la Organización Mundial de la Salud (OMS). Disponible en URLL

http://www.shef.ac.uk/FRAX/tool.jsp?lang=sp (último acceso 22/05/12).

El objetivo de identificar a las personas en riesgo debería ser asesorarlas en la prevención de caídas y cuando exista evidencia de que esta intervención es más beneficiosa que perjudicial y a un costo que la sociedad en la que viven pueda afrontar, ofrecerles tratamiento preventivo ${ }^{\$ \S \S \S}$ para las fracturas por fragilidad.

Vale destacar que en Argentina ha sido desarrollada y validada también una regla de predicción clínica por Ciapponi y col. ${ }^{25}$ en sendas muestras no probabilísticas de mujeres mayores de 45 años. Sólo requiere como insumo la edad y el peso de las pacientes, y permite identificar con un $95 \%$ de sensibilidad y un valor predictivo negativo de $98 \%$ a las mujeres con OP femoral. Ver tablas 2 y 3.
Tabla 2. Regla de predicción de osteoporosis densitométrica femoral con dos variables desarrollada en Buenos Aires (Argentina) por Ciapponi y col.

\begin{tabular}{l|c|c|c}
\multicolumn{2}{c|}{ Variable clínica } & $\begin{array}{c}\text { Asociación con osteoporosis } \\
\text { densitométrica OR (IC95\%) }\end{array}$ & Puntaje \\
\hline 1. Edad & 45 a 54 años & 1 & 0 \\
& 55 a 64 años & $3,58(1,92$ a 10,75$)$ & 4 \\
& 54 a 74 años & $7,29(2,37$ a 22,42) & 7 \\
\hline 2. Peso & Más de 75 años & $18,10(0,80$ a 6,43$)$ & 18 \\
& Más de $70 \mathrm{~kg}$ & 1 & 0 \\
& 60 a $69 \mathrm{~kg}$ & $2,33(0,92$ a 5,85$)$ & 2 \\
& Menos de $60 \mathrm{~kg}$ & $7,03(3,00$ a 16,44$)$ & 7 \\
\hline
\end{tabular}

Tabla 3. Riesgo predicho para osteoporosis femoral de acuerdo a la regla de Ciapponi y col.

\begin{tabular}{c|c|c} 
Puntaje & Riesgo aproximado de osteoporosis & \multirow{2}{*}{ Puntaje } \\
3 o menos & $<1,5 \%$ & \multirow{2}{*}{ N0 } \\
\hline 4 & 1,5 a $2,5 \%$ & \multirow{2}{*}{ Si } \\
\hline 5 a $\mathbf{~}$ & 4 a $9,9 \%$ & \\
\hline 11 a 13 & 10 a $15 \%$ & \\
\hline 14 a 20 & 20 a $25 \%$ & \\
\hline 20 0 más & Mayor a $25 \%$ &
\end{tabular}

\section{La problemática del sobre-rastreo de osteoporosis}

Como dijimos previamente, las principales Guías de Práctica Clínica acuerdan en no recomendar la realización de rastreo de osteoporosis antes de los 65 años de edad en pacientes que no presentan un riesgo aumentado de fractura osteoporótica, ya que antes de esta edad, la implementación de una estrategia de rastreo indiscriminada tiene muy baja costo-efectividad. Vale destacar el análisis económico realizado por Nguyen y col. ${ }^{26}$, basándose en datos de un meta-análisis, que evaluó que el costo estimado de evitar una fractura osteoporótica de cadera en la población general de mujeres post menopáusicas es de 120.000 libras esterlinas (US\$ 192.847). Ver en la tabla 4 el diferente rendimiento en términos del número de mujeres que deben ser rastreadas con densitometría osea para detectar un caso de osteoporosis pasible de tratamiento y eventualmente prevenir una fractura de cadera, en diferentes escenarios de acuerdo a la edad de comienzo de la pesquisa.

Tabla 4. Resultados hipotéticos a cinco años luego de aplicar una misma estrategia de rastreo en una cohorte de 10.000 mujeres blancas post menopáusicas a diferentes estratos de edad.

\begin{tabular}{|c|c|c|c|c|c|}
\hline \multirow[b]{2}{*}{ Puntaje } & \multicolumn{5}{|c|}{ Edad (años) } \\
\hline & 55 a 59 & 60 a 64 & 65 a 69 & 70 a 74 & 75 a 79 \\
\hline Prevalencia de osteoporosis por rango etario definida como un puntaje T menor a 2,5 & 0,0445 & 0,0650 & 0,1200 & 0,2025 & 0,2850 \\
\hline RR de fractura de cadera con tratamiento tomando comparado con el del grupo no tratado & \multicolumn{5}{|c|}{0,44 (IC95\% 0,18 a 0,97) } \\
\hline Casos de osteoporosis identificados cada 10.000 pacientes rastreados & 445 & 650 & 1200 & 2025 & 2850 \\
\hline Fracturas esperadas sin tratamiento $(24,50 \%)$ & 109 & 159 & 294 & 496 & 698 \\
\hline Fracturas de cadera esperadas con tratamiento $(1,25 \%)$ & 6 & 8 & 15 & 25 & 36 \\
\hline Fracturas de cadera prevenidas & 6 & 10 & 18 & 31 & 42 \\
\hline $\begin{array}{l}\text { Número de mujeres que es necesario rastrear en cada rango etario para prevenir una fractura } \\
\text { de cadera }\end{array}$ & 1667 & 1000 & 556 & 323 & 238 \\
\hline
\end{tabular}

Schnatz y col. determinaron que el $41,3 \%$ de las $\mathrm{DO}$ realizadas a mujeres post menopáusicas entre 2007 y 2009 en una institución privada de radiología situada en Hartford (capital del estado de Connecticut, Estados Unidos) eran inapropiadas respecto de los criterios diagnósticos imperantes en dicho momento histórico.

Esta percepción coincide con la de los autores de este artículo, que tenemos la impresión de que en Argentina se ha insta- lado en los medios de comunicación un discurso que sostiene que la osteoporosis es una condición clínica que debe comenzar a ser pesquisada apenas comienza la menopausia o aún durante la perimenopausia -o peor aún, que la menopausia misma es una especie de enfermedad-. Esto conduce a que una parte de nuestra población de mujeres sanas mayores de 45 años sea sometida a un "sobre-rastreo" con DO con el objetivo de pesquisar OP, con la consiguiente dilapidación de recur-

${ }^{5 \varsigma s \S 5}$ Hay diversos tratamientos disponibles pero la dificultad se encuentra en saber quien se verá beneficiado con su uso. 
sos (recursos económicos directos por el costo de dicha práctica, y recursos económicos indirectos como el tiempo empleado por la paciente para realizarla, el tiempo de la consulta dedicado a la prescripción, la explicación de los resultados, la tranquilización de la paciente respecto de algún resultado que le generó preocupación, etc.).
Otros riesgos potenciales de la sobreutilización de la DO son los resultados falsos positivos que suelen gatillar una prescripción innecesaria de tratamiento, la ansiedad de la paciente acerca del resultado, y la utilización del siempre escaso tiempo de la consulta que podría utilizarse para realizar otras prácticas preventivas importantes.

Recibido el 24/05/12 y aceptado el 04/06/12.

Referencias

1. Consensus Development Conference (1993) Diagnosis, prophylaxis and treatment of osteoporosis. Am J Med 94:646-650.

2. National Osteoporosis Foundation. Clinician's Guide to Prevention and Treatment of Osteoporosis. Washington, DC: National Osteoporosis Foundation; 2010.

3. Johnell O, Hertzman P. (2006). What evidence is there for the prevention and screening of osteoporosis?

Copenhagen, WHO Regional Office for Europe (Health Evidence Network report; http://www.euro.who.int/document/e88668.pdf, accessed 18 May 2006).

4. Kanis JA (2007) WHO Technical Report, University of Sheffield, UK: 66

5. Goeree R, O'Brien B, Pettitt D, et al. (1996) An assessment of the burden of illness due to osteoporosis in Canada. J Soc Obstet Gynaecol Can 18:15.

6. National Osteoporosis Foundation. America's Bone Health: The State of Osteoporosis and Low Bone Mass in Our Nation. Washington, DC: National Osteoporosis Foundation; 2002.

7. Diaz Curiel M, Garcia JJ, Carrasco JL, et al. (2001) Prevalencia de osteoporosis determinada por densitometría en la población femenina española. Med Clin (Barc) 116:86.

8. Clark P, Cons-Molina F, Deleze M, et al. (2009) The prevalence of radiographic vertebral fractures in Latin American countries: the Latin American Vertebral Osteoporosis Study (LAVOS). Osteoporos Int 20:275.

9. Schurman I, Bagur A, Claus-Hermberg H, et al. (2007) Guías para diagnóstico, prevención y tratamiento de la osteoporosis. Actual Osteol 3:117 and Rev Arg osteol 6:27.

10. Spivacow FR and Sanchez A (2010) Epidemiology, costs, and burden of osteoporosis in Argentina, 2009. Arch Osteoporos 5:1.

11. Burge RT, Dawson-Hughes B, Solomon D, Wong JB, King AB, Tosteson ANA. Incidence and economic burden of osteoporotic fractures in the United States, 2005-2025. J Bone Min Res. 11. Burge RT, Dawsor

12. Kanis JA, Jonsson B. Economic evaluation of interventions for osteoporosis. Osteoporosis International, 2002, 13(10):765-767.

13. Johnell O and Kanis JA (2006) An estimate of the worldwide prevalence and disability associated with osteoporotic fractures. Osteoporos Int 17:1726.

14. Norris RJ. Medical costs of osteoporosis. Bone 1992; 13(Suppl 2): S11-6.

15. Ström O, Borgström F, Kanis JA, Compston J, Cooper C, McCloskey EV, Jönsson B. Osteoporosis: burden, health care provision and opportunities in the EU. Arch Osteoporos (2011) 6:59-155.

16. Kanis JA, Melton LJ III, Christiansen C, Johnston CC, Khaltaev N. The diagnosis of osteoporosis. J Bone Miner Res. 1994:9(8):1137-1141.

Bolotin $\mathrm{HH}$, Sievanen $\mathrm{H}$. Inaccuracies inherent in dual-energy x-ray absorptiometry in vivo bone mineral density can seriously mislead diagnostic/prognostic interpretations of patient-specific bone fragility. J Bone Miner Res 2001;16:799-805

17. Jävinen TL, Sievänen H, Khan KM, Heinonen A, Kannus P. Shifting the focus in fracture prevention from osteoporosis to falls. BMJ 2008;336:124-6.

18. Nelson HD, Helfand M, Woolf SH, Allan JD. Screening for postmenopausal osteoporosis: a review of the evidence for the US Preventive Services Task Force. Ann Intern Med 2002;137:529-41.

19. Nguyen ND, Eisman JA, Nguyen TV. Anti-hip fracture efficacy of biophosphonates: a bayesian analysis of clinical trials. J Bone Miner Res 2006;21:340-9.

20. FRAX®: un nuevo instrumento para calcular el riesgo absoluto de fracturas a 10 años. Claus-Hermberg $\mathrm{H}$ et al .Medicina (Buenos Aires) 2009;69:571-575

21. FRAX@ y la evaluación de la probabilidad de fractura: introducción. Kanis J et al. Rev Metab Óseo y Min 2010;8(1):15-18.

22. FRAX-WHO fracture risk assessment tool. Avilable at: http://www.shef.ac.uk/FRAXI.

23. Screening for osteoporosis: U.S. Preventive Services Task Force Recommendation Statement. Ann Intern Med. 2011;154:356-364.

24. Ciapponi A y col. Regla de Predicción de Osteoporosis en Buenos Aires (PROBA): Desarrollo y Validación. Archivos de Medicina Familiar y General. Vol 5(1) 2007: 5-14. Disponible en URL http://archivos.famfyg.org/revista/index.php/amfyg/article/view/48 (último acceso 22/05/12).

25. Nguyen ND, Eisman JA, Nguyen TV. Anti-hip fracture efficacy of biophosphonates: a bayesian analysis of clinical trials. J Bone Miner Res 2006;21:340-9.

26. Schnatz PF, Marakovits KA, Dubois M, O'Sullivan DM. Osteoporosis screening and treatment guidelines: are they being followed?. Menopause. 2011;18(10):1072-8.

27. Moynihan R. Preventing overdiagnosis: how to stop harming the healthy. BMJ 2012;344:e3502 doi: 10.1136/bmj.e3502 (Published 29 May 2012). 